\title{
Liver biopsies from human females contain male hepatocytes in the absence of transplantation
}

\author{
Anne M Stevens ${ }^{1,2}$, W Michael McDonnell ${ }^{3}$, Meghan E Mullarkey ${ }^{1}$, Jennifer M Pang ${ }^{1}$, \\ Wendy Leisenring ${ }^{1}$ and J Lee Nelson ${ }^{1,3}$ \\ ${ }^{1}$ Fred Hutchinson Cancer Research Center, Seattle, Washington, USA; ${ }^{2}$ Department of Pediatrics and \\ ${ }^{3}$ Department of Medicine, University of Washington, Seattle, Washington, USA
}

\begin{abstract}
Fetal cells derived from pregnancy can persist in a woman's blood and tissues for decades and have been implicated in the pathogenesis of autoimmune disease. Transplantation studies based on donor sex mismatch suggest that circulating stem cells can lead to liver regeneration with donor-derived hepatocytes. However, male cells in female liver could derive from pregnancy. We investigated male cells in liver biopsies from women with sons and asked whether they were hematopoietic cells or hepatocytes. Fluorescence in situ hybridization for X- and Y-chromosomes with concomitant immunohistochemistry was employed to study 28 female liver biopsies: 14 with the autoimmune disease primary biliary cirrhosis (PBC), eight with Hepatitis $C$, and six with other diseases. Total male cells and those expressing hematopoietic (CD45) or hepatocyte (CAM-5.2) markers were quantified. None of the male cells were hematopoietic in origin, as shown by lack of CD45 expression. Instead, male cells with hepatocyte morphology expressing the hepatocyte marker CAM 5.2 were found in $25 \%$ of all biopsies (36\% of PBC and $14 \%$ of others). Overall, male cells were found in $36 \%$ of female liver biopsies. Of the PBC livers $43 \%$ had male cells compared to $25 \%$ of Hepatitis C biopsies and $33 \%$ of others. There was a trend toward increased numbers of male cells in PBC compared to others (mean 1 per 30000 host cells vs 0.17 in Hepatitis $C$ and $\mathbf{0 . 3 5}$ in others). Thus, male cells found in livers of women with sons include cells that express hepatocyte antigens. Therefore, transplantation and stem cell differentiation studies using sex difference to conclude that donor cells regenerate liver may be confounded by fetal microchimerism. Whether fetal cells play a role in autoimmune diseases like PBC merits further investigation.
\end{abstract}

Laboratory Investigation (2004) 84, 1603-1609, advance online publication, 25 October 2004; doi:10.1038/labinvest.3700193

Keywords: autoimmunity; fetal; liver; microchimerism; pregnancy; transplantation

Fetal cells traffic into the maternal circulation during pregnancy, where they can persist in blood and tissues for decades, creating a state of fetal microchimerism. ${ }^{1}$ Stem cell transplantation studies suggest that circulating hematopoietic stem cells can differentiate in the new host into parenchymal tissue cells, including liver. ${ }^{2}$ The majority of the evidence in humans is based upon finding male donor cells in female tissues. ${ }^{3-8}$ However, since male fetal cells may derive from prior pregnancies, male cells in stem cell transplantation recipients could also derive from the recipient's previous pregnancy with a male fetus.

Correspondence: Dr AM Stevens, MD, PhD, Immunogenetics, Fred Hutchinson Cancer Research Center, 1100 Fairview Ave N, D2-100, PO Box 19024, Seattle, WA 98109, USA.

E-mail: astevens@fhcrc.org

Received 15 June 2004; revised 13 September 2004; accepted 14 September 2004; published online 25 October 2004
Fetal microchimerism has been implicated in the pathogenesis of some autoimmune diseases, and several lines of evidence suggest that fetal cells could play a role in the pathogenesis of primary biliary cirrhosis (PBC). PBC is an autoimmune disease characterized by periportal inflammation directed at bile duct epithelia, with resultant loss of interlobular and septal bile ducts leading to cholestasis. ${ }^{9}$ Bile duct loss is associated with two other diseases in which both host and nonhost cells are found within the liver, chronic graft-vs-host disease (GVHD) following stem cell transplantation and liver allograft rejection. ${ }^{10-13}$ PBC occurs predominantly in individuals most likely to host fetal chimeric cells: women in the postreproductive years. ${ }^{9}$ Moreover, PBC patients have been reported to have higher numbers of pregnancies compared to friend controls. ${ }^{14} \mathrm{~A}$ few studies have reported fetal cells and DNA in liver samples from PBC patients, but most also found fetal microchimerism in control 
livers. ${ }^{15-18}$ The phenotypes of fetal cells in the liver could potentially affect whether they contribute to disease, but have not been explored.

We investigated male (presumed fetal) cells in liver biopsies from women with PBC and other diseases to determine whether identified cells were hematopoietic or hepatocytes.

\section{Materials and methods}

\section{Patients}

Caucasian patients who had liver biopsies as part of routine medical care were recruited prospectively from the Seattle area. Only women who had given birth to sons were included because of the assay used, which identifies male cells in female tissue. One patient had two biopsies on two different dates. The Fred Hutchinson Cancer Research Center and University of Washington Human Subjects Committees approved the protocol, information gathering, and consent forms. Informed consent was obtained from all patients.

\section{Immunohistochemistry (IHC)}

In order to characterize the male cells in female liver tissue, a method was developed by which multiple phenotypic markers could be tested simultaneously with $\mathrm{X}$ - and Y-chromosome markers in the same cells in the same tissue section, as previously reported. ${ }^{19}$ Conditions were optimized for each sample to allow for maintenance of the IHC signal during the fluorescence in situ hybridization (FISH) procedure. Tissue sections were first subjected to two-color immunohistochemistry with two different specific antibodies; the same section was then probed for X- and Y-chromosomes. Photographs were taken after both procedures had been performed. In detail, formalin-fixed, paraffin-embedded liver needle biopsies were cut into $4 \mu \mathrm{m}$ thick sections onto charged capillary gap slides. After removing the paraffin with xylene washes and serial rehydration, antigen retrieval was performed with Target Retrieval Solution. (DAKO, Carpinteria, CA, USA). The solution was applied between two slides by capillary action, and the slides were exposed to steam in a vegetable steamer for $40 \mathrm{~min}$. IHC was then performed using the ABC system according to the manufacturer's protocol (Vector Laboratories, Burlingame, CA, USA). First, IHC was performed with antibodies to CD45 specific for hematopoietic cells (mouse monoclonal antibody, DAKO). After application of secondary reagents and development with the substrate DAB (BioFX Laboratories, Owings Mills, MD, USA), tissues were treated with an avidin-biotin blocking kit (Vector Laboratories). To label endothelial cells, antibody to Von Willebrand Factor antibody (VWF, DAKO) was used. Steam antigen retrieval was repeated and signals deve- loped with the ABC system and SG substrate (Vector). For labeling hepatocytes, steam antigen retrieval was repeated and tissues were treated with pepsin $(100 \mu \mathrm{g} / \mathrm{ml})$ for $10 \mathrm{~min}$ (Sigma, St Louis, MO, USA) followed by an antibody specific for cytokeratins (CAM 5.2, Becton Dickinson Immunocytometry Systems, San Jose, CA, USA). The hepatocyte signals were developed with the ABC system and SG substrate (Vector). Studies with no primary antibody were performed as negative controls. No signals were visible on these slides.

\section{FISH for X-and Y-Chromosomes}

Following IHC, tissues were treated with $0.2 \mathrm{~N} \mathrm{HCl}$ for $20 \mathrm{~min}, 2 \times \mathrm{SSC}$ for $30 \mathrm{~min}$ at $80^{\circ} \mathrm{C}$, Proteinase $\mathrm{K}$ (50-100 $\mu \mathrm{g} / \mathrm{ml}$, Invitrogen Life Technologies, Carlsbad, CA, USA) at $37^{\circ} \mathrm{C}$ for $10 \mathrm{~min}$, rinsed, and then fixed in $4 \%$ formaldehyde/PBS. After dehydration, the sections were probed with DNA probes specific for DXS1 labeled with orange fluorescent Cy3-dUTP (Pharmacia Biotech UK Limited), and for DYS1 labeled with green fluorescent fluorescein-12-dUTP (Roche Applied Science, Germany). Probes and genomic DNA were denatured on the slides at $90^{\circ} \mathrm{C}$ for $5 \mathrm{~min}$, incubated overnight at $37^{\circ} \mathrm{C}$, and washed with $50 \%$ formamide $/ 2 \times$ SSC and $2 \times$ SSC at $42^{\circ} \mathrm{C}$. Male control tissue was hybridized during each experiment. The efficiency of Y-chromosome detection by hybridization of $4 \mu \mathrm{m}$ sections was $86 \%$, as demonstrated on male liver control tissue. Negative controls were liver samples from females 8 and 24 months old with no history of transfusions. Sections from these subjects constituting a total surface area of $6 \mathrm{~cm}^{2}$ was assayed, and no male cells were found. In order to normalize the frequency of male cells in each tissue sample, a method was developed to estimate the number of total host cells per tissue section. All of the nuclei with two sex chromosomes were counted and the surface area of tissue measured on each of three slides. On the average, there were 1300 nuclei with two sex chromosomes visible per $\mathrm{mm}^{2}$ of tissue. For the patient samples, tissue sections ranging from 1 to $33 \mathrm{~mm}^{2}$ (an average of $25 \mathrm{~mm}^{2}$ of tissue per subject) were analyzed.

\section{Statistical Analysis}

The number of subjects studied was determined based on preliminary data from our laboratory and the literature to give a power of $80 \%$ to detect a difference of 8 cells $/ \mathrm{cm}^{2}$ between PBC patients and controls. For all statistical analyses, results from only the first biopsy sample available for the PBC patient with two biopsies was utilized. Univariable comparisons of continuously valued demographic factors and cell numbers were made using a Wilcoxon rank-sum test. Dichotomous factors were compared using Fisher's exact test. Comparisons of cell counts adjusted for age at biopsy were carried 
out using linear regression models with dichotomous covariates for case vs control status and for age $<40$ vs age $\geq 40$ years. All reported $P$-values are two sided.

\section{Results}

\section{Study Subjects}

The clinical characteristics of the patients are shown in Table 1. In total, 28 subjects were chosen for the study based on their histories of having previously delivered male children, so that FISH for X- and Ychromosomes could be used to detect male cells in female tissues. The subjects were divided between those with PBC (14 subjects) and other diagnoses (14 subjects). There were no significant differences between PBC patients and other patients in the median number of total children (2) or the number of sons (1) born to each subject. PBC patients were older than other subjects (median 56 vs 43.5 years, $P=0.002$ ), attributed to PBC occurring in middle age, whereas many of the other subjects had
Hepatitis C, often developing disease earlier in life. Histological data was derived from reports by local hospital pathologists. For PBC, liver biopsy reports were classified as either stage 1-2 or stage 3-4. Because there are discrepancies between the Ludwig and the Scheuer classification systems ${ }^{20,21}$ the presence of bridging fibrosis was used as the index criterion to distinguish stage 3-4 (five subjects) from stage 1-2 (eight subjects).

\section{Phenotypes of Male Cells in Liver}

To determine the phenotypes of the male cells in liver, simultaneous IHC and FISH for X- and Ychromosomes were performed with antibodies to cytokeratin (CAM 5.2), which has been shown to be specific in the liver for hepatocytes and biliary epithelial cells. ${ }^{4}$ To identify hematopoietic microchimeric cells, tissues were costained with antibody specific for CD45, which is expressed on all leukocytes, including Kupffer cells. ${ }^{22}$ Surprisingly, no male cells expressed CD45. However, many ex-

Table 1 Clinical characteristics of patients

$\begin{array}{lccc}\text { Subject } & \text { Age at BX } & \text { Number of pregnancies } \\ \text { (sons) } & \begin{array}{c}\text { Last son to Bx } \\ \text { (years) }\end{array} & \text { Liver histology }\end{array}$

\section{(a) Characteristics of subjects}

PBC patients

\begin{tabular}{|c|c|c|c|c|c|}
\hline 1 & 32 & $3(2)$ & 3,4 & Stage 1-2 & $\mathrm{AMA}+$ \\
\hline 2 & 59,61 & $2(1)$ & 32,34 & Stage 1-2 & AMA- \\
\hline 3 & 62 & 4 (1) & 36 & Stage $1-2$ & AMA+ \\
\hline 4 & 54 & 8 (1) & 19 & Stage 3-4 & AMA+ \\
\hline 5 & 59 & $3(3)$ & 33 & Stage 1-2 & AMA+ \\
\hline 6 & 53 & $2(1)$ & $\mathrm{nk}$ & Stage 1-2 & AMA+ \\
\hline 7 & 57 & $2(1)$ & 38 & Stage $3-4$ & AMA+ \\
\hline 8 & 52 & $2(1)$ & 30 & Stage $1-2$ & AMA+ \\
\hline 9 & 58 & $2(1)$ & 31 & Stage 3-4 & AMA+ \\
\hline 10 & 52 & $2(1)$ & 31 & nk & AMA+ \\
\hline 11 & 44 & $2(1)$ & 28 & Stage 1-2 & AMA+ \\
\hline 12 & 58 & $2(1)$ & 28 & Stage 3-4 & AMA+ \\
\hline 13 & 60 & $4(3)$ & 37 & Stage 3-4 & AMA+ \\
\hline 14 & 55 & $2(2)$ & 32 & Stage 1-2 & AMA- \\
\hline \multicolumn{6}{|l|}{ (b) Others (Dx) } \\
\hline 1 (Steatosis) & 43 & $1(1)$ & nk & Steatosis & nk \\
\hline $2(\mathrm{HCV})$ & 44 & $3(2)$ & nk & $\begin{array}{l}\text { Piecemeal necrosis and lobular } \\
\text { inflammation }\end{array}$ & $\mathrm{nk}$ \\
\hline 3 (steatohepatitis) & 54 & $1(1)$ & 26 & Steatohepatitis with cirrhosis & ANA+ \\
\hline 4 (HCV) & 36 & $1(1)$ & 21 & $\mathrm{CAH}$ & ANA- \\
\hline 5 (HCV) & 38 & $3(3)$ & 7 & Mild chronic hepatitis & nk \\
\hline 6 (Normal) & 59 & $2(1)$ & 35 & Chronic active portal inflammation & nk \\
\hline 7 (AIH) & 26 & $1(1)$ & 2 & Chronic hepatitis & $\begin{array}{l}\text { ANA+ } \\
\text { ASMA+ }\end{array}$ \\
\hline 8 (steatosis) & 32 & $5(1)$ & 17 & Fatty metamorphosis & ANA- \\
\hline 9 (HCV) & 45 & $3(3)$ & 24 & CAH & nk \\
\hline 10 (HCV) & 32 & $3(2)$ & 10 & $\mathrm{CAH}$ & ANA- \\
\hline 11 (HCV) & 45 & $1(1)$ & nk & Mild CAH; diffuse, mild fatty changes & nk \\
\hline 12 (HCV) & 44 & $2(1)$ & 22 & CAH; mild fibrous portal expansion & ANA- \\
\hline 13 (HCV) & 48 & $2(2)$ & 22 & $\mathrm{CAH}$ & nk \\
\hline 14 (steatosis) & 52 & $\geq 1(\geq 1)$ & nk & Chronic hepatitis, fatty changes & nk \\
\hline
\end{tabular}

Bx, biopsy; nk, not known; AMA+, with antimitochondrial antibodies; AMA-, no antimitochondrial antibodies; Dx, diagnosis; Abs, antibodies; HCV, Hepatitis C Virus; AIH, autoimmune hepatitis; CAH, chronic active hepatitis; ANA+, with antinuclear antibodies; ANA-, no antinuclear antibodies; ASMA+, with antismooth muscle antibodies. 

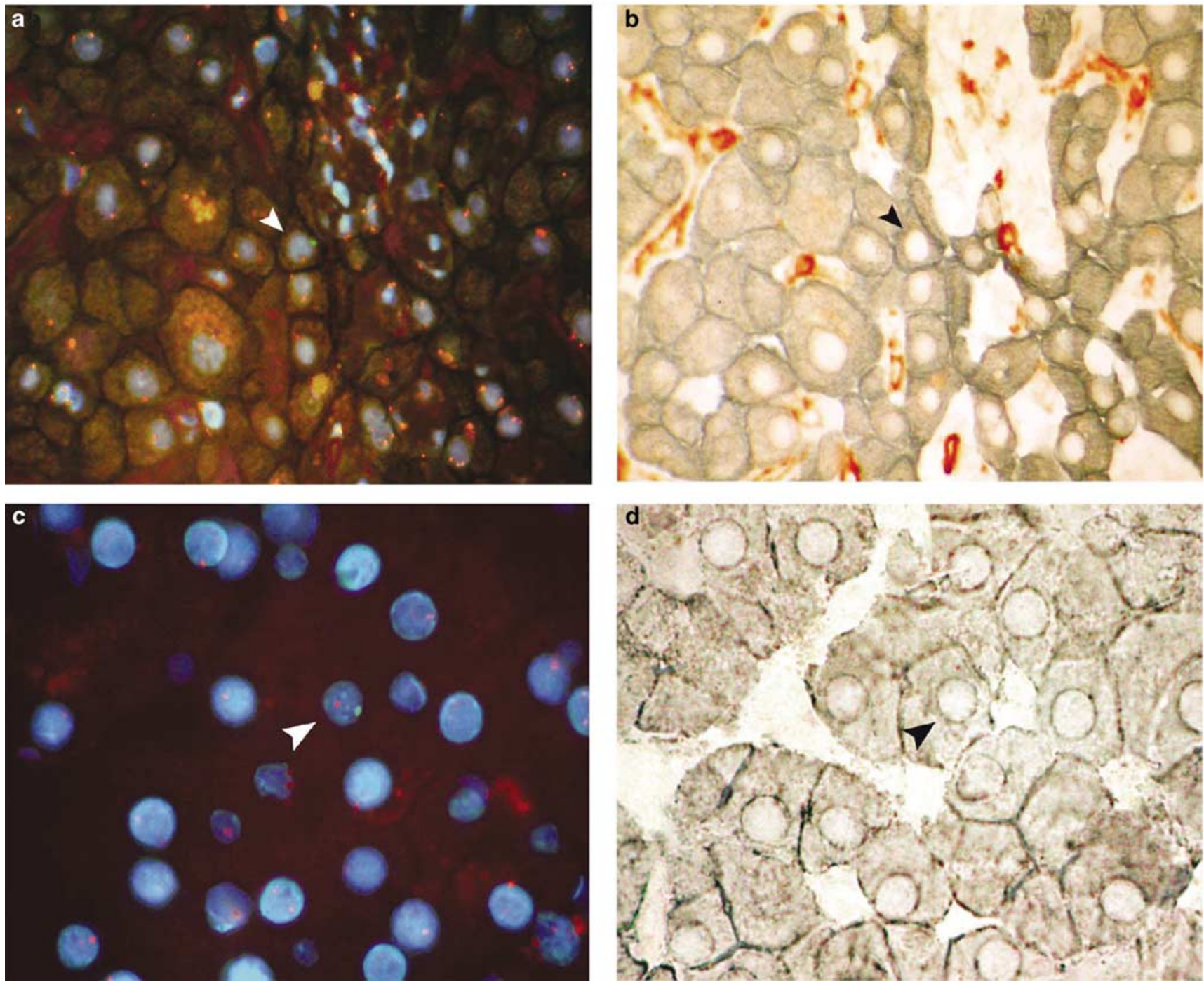

Figure 1 Male (fetal) hepatocytes in liver tissue from women with sons. (a) Fluorescence microscopy of liver from a woman with PBC containing a male cell $(\times 100)$. Cell nuclei are identified with DAPI (blue). The arrowhead designates a male nucleus containing one green Y-chromosome signal and one red X-chromosome signal. The host nuclei contain two red X-chromosome signals. (b) Light microscopy of the same section of tissue as in (a) $(\times 100)$. The gray substrate identifies CAM 5.2 expression on hepatocytes. The brown substrate identifies CD45 expression on hematopoietic cells. The same male cell shown in (a) expresses CAM 5.2 and does not express CD45 (arrowhead). The male cell is also morphologically similar to the surrounding hepatocytes. (c) Fluorescence microscopy of liver from a control subject containing a male cell $(\times 100)$. Arrowhead designates a male nucleus. (d) Light microscopy of the same section of tissue as in $(\mathbf{c})(\times 100)$. This male cell is also morphologically similar to the surrounding hepatocytes and does not express CD45. (There are no CD45 + cells in this section of tissue).

pressed the hepatocyte marker and morphologically resembled hepatocytes (Figure 1). Costaining with CD45 also served as an internal control in that the lack of CD45 expression demonstrated that the observed male hepatocytes were not male hematopoietic cells overlying host hepatocytes. Male hepatocytes were found in $25 \%$ of all subjects (Table 2). Five PBC biopsies contained male hepatocytes (36\%) compared to two with alternate diagnoses (one with Hepatitis C, one with steatosis, $14 \%)(P=0.19)$. Although in different sections of tissues hyperproliferative bile duct epithelia staining strongly for cytokeratins were observed, no male cells were found within the bile ducts. Male cells were not found in areas of inflammation either, as identified by CD45 expression as well as hematoxylin staining of adjacent sections.

In all, $62 \%$ of the male cells identified expressed neither CD45 nor CAM5.2. In one sample (the second biopsy from PBC patient 2), a cluster of male cells was identified. The cells were seen in two different sections and totaled 45 male cells. These cells stained with neither CAM5.2 nor CD45, and did not appear to have morphology consistent with either hepatocytes or hematopoietic cells.

To determine if the uncharacterized male cells were endothelial cells, 25 sections from six subjects 
Table 2 Male cells identified in liver tissue

(a) PBC patients $(\mathrm{N}=14)$

\begin{tabular}{llrlllllllllllll}
\multicolumn{1}{c}{1} & $2 a^{\mathrm{a}}$ & $2 b$ & 3 & 4 & 5 & 6 & 7 & 8 & 9 & 10 & 11 & 12 & 13 & 14 & Mean \\
1.29 & 3.9 & 99 & 0 & 0 & 0 & 0 & 0 & 1.35 & 0 & 1.14 & 0 & 4.6 & 1.71 & 0 & 1.0 \\
1.4 & 4.18 & 107 & 0 & 0 & 0 & 0 & 0 & 1.5 & 0 & 1.25 & 0 & 5 & 1.85 & 0 & 1.08 \\
0.475 & 4.18 & 0 & 0 & 0 & 0 & 0 & 0 & 0 & 0 & 0.63 & 0 & 0.83 & 0.93 & 0 & 0.50 \\
0.95 & 0 & 107 & 0 & 0 & 0 & 0 & 0 & 1.45 & 0 & 0.63 & 0 & 4.2 & 0.93 & 0 & 0.58
\end{tabular}

(b) Other patients $(\mathrm{N}=14)$

\begin{tabular}{lllllllllllllllll} 
& 1 & 2 & 3 & 4 & 5 & 6 & 7 & 8 & 9 & 10 & 11 & 12 & 13 & 14 & Mean \\
\hline Male cells per 30000 host cells & 1.59 & 0 & 0 & 1.02 & 0 & 0 & 0.54 & 0 & 0 & 0 & 0.4 & 0 & 0 & 0 & 0.24 & 0 \\
Male cells per 25 mm & 1.73 & 0 & 0 & 1.1 & 0 & 0 & 0.58 & 0 & 0 & 0 & 0.38 & 0 & 0 & 0 & 0.28 & 0 \\
\# Male hepatocytes per 25 mm & 1.73 & 0 & 0 & 1.08 & 0 & 0 & 0 & 0 & 0 & 0 & 0 & 0 & 0 & 0 & 0.2 \\
\# Male nonhepatocytes per $25 \mathrm{~mm}^{2}$ & 0 & 0 & 0 & 0 & 0 & 0 & 0.58 & 0 & 0 & 0 & 0.38 & 0 & 0 & 0 & 0.07 \\
\hline
\end{tabular}

${ }^{\mathrm{a} O n l y}$ the first biopsy from patient 2 was used for statistical analysis.

were stained for the endothelial cell marker Von Willebrand factor (VWF) along with CD45. None of the male cells identified in these samples expressed VWF or CD45. Thus, in the samples studied, fetal endothelial cells were not found.

\section{Total Male Cells (Hepatocytes and Nonhepatocytes) in PBC Compared to Other Diseases}

Overall, male cells were found in $36 \%$ of female liver biopsies (Table 2). There was a trend toward higher prevalence and fraction of male cells in livers from PBC patients when compared to controls. Male cells were observed in $43 \%$ of $\mathrm{PBC}$ and $29 \%$ of control biopsies $25 \%$ of Hepatitis C, $33 \%$ of others, $P=0.70$ ). In PBC livers, male cells were observed at a level of 1.0 per 30000 host cells $\left(1.08\right.$ per $25 \mathrm{~mm}^{2}$, range $0-5)$. In comparison, other livers had 0.24 per $30000\left(0.3\right.$ per $25 \mathrm{~mm}^{2}$, range $\left.0-1.73\right)$. Hepatitis C patients had the lowest rate of male microchimerism, 0.17 per 30000 host cells $\left(0.18\right.$ per $25 \mathrm{~mm}^{2}$, range 0-1.0). Thus, there was a trend toward increased numbers of male cells in PBC, but neither the proportion of subjects with male cells $(P=0.70)$ nor the levels $(P=0.22)$ were statistically significantly higher than in other diseases. The difference in cell frequency that we observed between PBC and other diseases $\left(0.8\right.$ cells $\left./ 25 \mathrm{~mm}^{2}\right)$ was smaller than the difference detectable with $80 \%$ power in this study $\left(2\right.$ cells $\left./ 25 \mathrm{~mm}^{2}\right)$. Thus, there may be an increase in male (fetal) cells in PBC, but a larger study is required to test the possibility.

There was no correlation between the presence of male cells and the number of sons each subject had delivered or the time elapsed since the birth of the most recent son. Neither the presence of antimitochondrial antibodies nor histological stage of disease were associated with male microchimerism, although the number of subjects in each subgroup was small.

PBC patients were older than the control subjects. Because it is not known if age affects the persistence of fetal microchimerism, an analysis was carried out to adjust for age at biopsy. Subjects were categorized into two groups: $<40$ vs $\geq 40$ years of age. A covariate was included for a linear regression model with PBC vs other diseases as predictors for each of the cell type outcome. Adjusting for age did not affect the association between underlying disease and frequency of either fetal hepatocytes or nonhepatocytes in the liver.

\section{Discussion}

In this study, rare male cells of presumed fetal origin were identified in the liver in women who had never received a stem cell or organ transplant. Many of the male cells morphologically resembled hepatocytes and expressed a surface marker for hepatocytes.

Previous reports suggest that after stem cell transplantation donor cells may engraft into the liver and transform into hepatocytes, biliary epithelial cells, and endothelial cells at a frequency as high as $7 \%$ in the period following transplantation. ${ }^{3-8,23-25}$ In the above studies, the pregnancy histories of the stem cell transplantation patients or donors were not reported, with one exception that we know of (response to ${ }^{26}$ ). Therefore, the male cells found in female liver could have represented fetal cells from prior pregnancies. In previous work, we demonstrated that maternal cells can also be found in the liver. ${ }^{19}$ Thus, transplant studies identifying female (presumed donor) cells in male tissues could also potentially be confounded by maternal microchimerism.

How circulating fetal cells might lead to cells in liver expressing a hepatocyte phenotype is not 
known. Transdifferentiation of hematopoietic cells into somatic cells has been reported, but is controversial. ${ }^{2}$ Another possibility is fusion between fetal cells and host hepatocytes ${ }^{23,25,27-29}$ and reviewed in Alison et al, ${ }^{30}$ although transformation has been demonstrated to occur without fusion..$^{24,31}$ In the case of male fetal cells fusing with maternal hepatocytes, we would expect to find polyploid nuclei with XXXY or XXY karyotypes apparent in the tissue sections assayed. In our study, female polyploid cells were common, and thus multiple sex chromosomes per nucleus were detectable in the $4 \mu \mathrm{m}$-thick sections. However, we did not observe any male polyploid nuclei, making fusion less likely. However, because of the presence of partial nuclei in some tissue sections, undetected products of fetal-maternal fusion remains a formal possibility.

The fetal hepatocyte phenomenon was not specific for the autoimmune disease PBC, although there was a trend toward higher prevalence and numbers in PBC patients. That many of the controls had chronic inflammatory diseases such as Hepatitis C, but carried no fetal cells suggests that increased fetal microchimerism may not be merely a nonspecific response to inflammation, but may be amplified in autoimmune disease. Likewise, fetal cells could be amplified in graft rejection or GVHD. The levels of male cells in our study were similar to those found in most previous studies of PBC livers. ${ }^{15,16}$

In one other report partial characterization of the male cells in liver from PBC patients showed that most were hematopoietic, expressing CD45 and HLA-DR. ${ }^{17}$ In our study, no male cells expressed CD45, and male cells were not found in the inflammatory infiltrates surrounding the bile ducts, suggesting that they were not inflammatory cells participating in disease pathogenesis. Since biliary epithelial cells are thought to be the immune targets for PBC, that no fetal biliary epithelial cells were found suggests that the fetal cells do not serve as immune targets in PBC. Interestingly, many of the fetal cells expressed neither CD45, VWF, nor cytokeratins. These cells may be fetal stellate cells (specialized myofibroblasts) or another unknown cell type. The biological role of these uncharacterized fetal cells is an area of active investigation.

It was presumed in this study, as in previous studies, that the male cells found in the female tissues were derived from a former fetus. There are other potential, though less likely sources of male cells. Since blood transfusions have been shown to lead to long-lasting microchimerism, the male cells detected in female patients could theoretically be of blood donor origin. ${ }^{32}$ The contribution of blood transfusion to tissue microchimerism is not known. Three of the control patients received transfusions, but none of those patients had male cells in their livers. Only one of the PBC patients had a trans- fusion (male cells were found in the liver of this patient). Therefore, transfusion could not have made a major contribution to male microchimerism in the overall study. Twin/twin transfusion is another possible source of microchimerism. The subjects in this study had no known twins, but male cells could be derived from an unrecognized male 'vanishing twin' early in gestation. ${ }^{33}$ We conclude that the male cells detected in our subjects were most likely to be of fetal origin. Definitive evidence for the source of male cells could be obtained by detection of noninherited, nonshared fetal alleles of polymorphic genes, as has been employed recently in blood samples. ${ }^{34}$

In summary, our data support the concept that fetal cells, known to persist in pregnant women long after pregnancy, can take the form of hepatocytes. Fetal microchimerism in the liver may be a common occurrence and may or may not be associated with disease. Studies of microchimerism in transplantation based solely on sex mismatch thus should be interpreted with caution and preferably conducted with methods that can rule out confounding by fetal and/or maternal microchimerism. Whether fetal hepatocytes could act as immune targets or as a source of foreign antigen leading to chronic inflammatory diseases like PBC is not known. Fetal cells could also contribute to an alternative mechanism of pathogenesis, altering the host response to normal environmental pathogens. ${ }^{35}$ Finally, fetal cells in the normal liver could contribute to the induction of tolerance to fetal antigens that allows the maternal immune system to overlook microchimeric fetal cells, both during the pregnancy and for years after. $^{36}$ The biological effects of fetal microchimerism in maternal liver merit further study.

\section{Acknowledgements}

We thank Jennifer Brackensick for patient coordination and recruitment, Dr Margaret Shuhart and Dr George McDonald for recruiting patients, and Dr Joseph Siebert for assistance in sample collection. The study was financially supported by NIH AI-45952, AI-41721, AI-45659.

\section{References}

1 Nelson JL. Microchimerism: incidental byproduct of pregnancy or active participant in human health? Trends Mol Med 2002;8:109-113.

2 Herzog EL, Chai L, Krause DS. Plasticity of marrowderived stem cells. Blood 2003;102:3483-3493.

3 Alison MR, Poulsom R, Jeffery R, et al. Hepatocytes from non-hepatic adult stem cells. Nature 2000; 406:257.

4 Theise ND, Nimmakayalu M, Gardner R, et al. Liver from bone marrow in humans. Hepatology 2000;32: 11-16. 
5 Gao Z, McAlister VC, Williams GM. Repopulation of liver endothelium by bone-marrow-derived cells. Lancet 2001;357:932-933.

6 Fogt F, Beyser KH, Poremba C, et al. Recipient-derived hepatocytes in liver transplants: a rare event in sexmismatched transplants. Hepatology 2002;36:173-176.

7 Okamoto R, Yajima T, Yamazaki M, et al. Damaged epithelia regenerated by bone marrow-derived cells in the human gastrointestinal tract. Nat Med 2002; 8:1011-1017.

8 Korbling M, Katz RL, Khanna A, et al. Hepatocytes and epithelial cells of donor origin in recipients of peripheral-blood stem cells. N Engl J Med 2002; 346:738-746.

9 Talwalkar JA, Lindor KD. Primary biliary cirrhosis. Lancet 2003;362:53-61.

10 Czaja AJ. Chronic graft-versus-host disease and primary biliary cirrhosis: sorting the puzzle pieces. Lab Invest 1994;70:589-592.

11 Wiesner RH, Batts KP, Krom RA. Evolving concepts in the diagnosis, pathogenesis, and treatment of chronic hepatic allograft rejection. Liver Transpl Surg 1999;5:388-400.

12 Quaranta S, Shulman H, Ahmed A, et al. Autoantibodies in human chronic graft-versus-host disease after hematopoietic cell transplantation. Clin Immunol 1999;91:106-116.

13 Inomata Y, Tanaka K. Pathogenesis and treatment of bile duct loss after liver transplantation. J Hepatobiliary Pancreat Surg 2001;8:316-322.

14 Parikh-Patel A, Gold E, Utts J, et al. The association between gravidity and primary biliary cirrhosis. Ann Epidemiol 2002;12:264-272.

15 Tanaka A, Lindor K, Gish R, et al. Fetal microchimerism alone does not contribute to the induction of primary biliary cirrhosis. Hepatology 1999;30: 833-838.

16 Corpechot C, Barbu V, Chazouilleres O, et al. Fetal microchimerism in primary biliary cirrhosis. J Hepatol 2000;33:696-700.

17 Fanning PA, Jonsson JR, Clouston AD, et al. Detection of male DNA in the liver of female patients with primary biliary cirrhosis. J Hepatol 2000;33:690-695.

18 Invernizzi P, De Andreis C, Sirchia SM, et al. Blood fetal microchimerism in primary biliary cirrhosis. Clin Exp Immunol 2000;122:418-422.

19 Stevens AM, Hermes HM, Rutledge JC, et al. Myocardial-tissue-specific phenotype of maternal microchimerism in neonatal lupus congenital heart block. Lancet 2003;362:1617-1623.

20 Scheuer PJ. Ludwig Symposium on biliary disorderspart II. Pathologic features and evolution of primary biliary cirrhosis and primary sclerosing cholangitis. Mayo Clin Proc 1998;73:179-183.

21 Ludwig J. Etiology of biliary cirrhosis: diagnostic features and a new classification. Zentralbl Allg Pathol 1988;134:132-141.

22 Prickett TC, McKenzie JL, Hart DN. Characterization of interstitial dendritic cells in human liver. Transplantation 1988;46:754-761.

23 Vassilopoulos G, Wang PR, Russell DW. Transplanted bone marrow regenerates liver by cell fusion. Nature 2003;422:901-904.

24 Harris RG, Herzog EL, Bruscia EM, et al. Lack of a fusion requirement for development of bone marrowderived epithelia. Science 2004;305:90-93.

25 Willenbring $\mathrm{H}$, Bailey AS, Foster M, et al. Myelomonocytic cells are sufficient for therapeutic cell fusion in liver. Nat Med 2004;10:744-748.

26 McDonnell WM, Nelson JL. Liver stem cells from bone marrow (Letter). Hepatology 2000;32:1181.

27 Terada N, Hamazaki T, Oka M, et al. Bone marrow cells adopt the phenotype of other cells by spontaneous cell fusion. Nature 2002;416:542-545.

28 Ying QL, Nichols J, Evans EP, et al. Changing potency by spontaneous fusion. Nature 2002;416:545-548.

29 Wang X, Willenbring H, Akkari Y, et al. Cell fusion is the principal source of bone-marrow-derived hepatocytes. Nature 2003;422:897-901.

30 Alison MR, Poulsom R, Otto WR, et al. Recipes for adult stem cell plasticity: fusion cuisine or readymade? J Clin Pathol 2004;57:113-120.

31 Newsome PN, Johannessen I, Boyle S, et al. Human cord blood-derived cells can differentiate into hepatocytes in the mouse liver with no evidence of cellular fusion. Gastroenterology 2003;124:1891-1900.

32 Lee TH, Paglieroni T, Ohto H, et al. Survival of donor leukocyte subpopulations in immunocompetent transfusion recipients: frequent long-term microchimerism in severe trauma patients. Blood 1999;93:3127-3139.

33 Landy HJ, Keith LG. The vanishing twin: a review. Hum Reprod Update 1998;4:177-183.

34 Lambert NC, Erickson TD, Yan Z, et al. Quantification of maternal microchimerism by HLA-specific real-time polymerase chain reaction: studies of healthy women and women with scleroderma. Arthritis Rheum 2004; 50:906-914.

35 Selmi C, Balkwill DL, Invernizzi P, et al. Patients with primary biliary cirrhosis react against a ubiquitous xenobiotic-metabolizing bacterium. Hepatology 2003; 38:1250-1257.

36 Palmer JM, Kirby JA, Jones DE. The immunology of primary biliary cirrhosis: the end of the beginning? Clin Exp Immunol 2002;129:191-197. 